Cochrane Database of Systematic Reviews

\title{
Heated tobacco products for smoking cessation and reducing smoking prevalence (Protocol)
}

Tattan-Birch H, Jackson S, Shahab L, Hartmann-Boyce J, Kock L, Simonavicius E, Brose L, Brown $\mathrm{J}$

Tattan-Birch H, Jackson S, Shahab L, Hartmann-Boyce J, Kock L, Simonavicius E, Brose L, Brown J. Heated tobacco products for smoking cessation and reducing smoking prevalence (Protocol).

Cochrane Database of Systematic Reviews 2020, Issue 11. Art. No.: CD013790.

DOI: 10.1002/14651858.CD013790.

www.cochranelibrary.com 
TABLE OF CONTENTS

HEADER 1

ABSTRACT

BACKGROUND

OBJECTIVES

METHODS

ACKNOWLEDGEMENTS

REFERENCES

APPENDICES

HISTORY

CONTRIBUTIONS OF AUTHORS

DECLARATIONS OF INTEREST

SOURCES OF SUPPORT 
[Intervention Protocol]

\section{Heated tobacco products for smoking cessation and reducing smoking prevalence}

Harry Tattan-Birch ${ }^{1}$, Sarah Jackson ${ }^{1}$, Lion Shahab ${ }^{1}$, Jamie Hartmann-Boyce ${ }^{2}$, Loren Kock ${ }^{1}$, Erikas Simonavicius ${ }^{3}$, Leonie Brose ${ }^{3}$, Jamie Brown ${ }^{1}$

1Department of Behavioural Science and Health, University College London, London, UK. ${ }^{2}$ Nuffield Department of Primary Care Health Sciences, University of Oxford, Oxford, UK. ${ }^{3}$ Department of Addictions, Institute of Psychiatry, Psychology \& Neuroscience, King's College London, London, UK

Contact address: Jamie Brown, jamie.brown@ucl.ac.uk.

Editorial group: Cochrane Tobacco Addiction Group.

Publication status and date: New, published in Issue 11, 2020.

Citation: Tattan-Birch H, Jackson S, Shahab L, Hartmann-Boyce J, Kock L, Simonavicius E, Brose L, Brown J. Heated tobacco products for smoking cessation and reducing smoking prevalence (Protocol). Cochrane Database of Systematic Reviews 2020, Issue 11. Art. No.: CD013790. DOI: 10.1002/14651858.CD013790.

Copyright ( 2020 The Cochrane Collaboration. Published by John Wiley \& Sons, Ltd.

\section{A B S T R A C T}

\section{Objectives}

This is a protocol for a Cochrane Review (intervention). The objectives are as follows:

To evaluate the effectiveness and safety of HTPs for smoking cessation and the impact of HTPs on smoking prevalence. 


\section{B A C K G R O U N D}

\section{Description of the condition}

Tobacco use kills 8 million people each year, making it the leading preventable cause of death worldwide (Drope 2018). Approximately $90 \%$ of these deaths result from the most harmful form of tobacco consumption: smoking (Drope 2018). Reducing smoking prevalence is therefore one of the most effective ways of improving population health (Holford 2014).

Although most smokers want to quit, smoking is highly addictive and most who try fail, with less than $10 \%$ still abstinent a year after making a serious attempt to stop (Jackson 2019a). Available treatments such as behavioural support, varenicline, and nicotine replacement therapy (NRT) improve the chance that these attempts will succeed (Cahill 2016; Hartmann-Boyce 2018; Hartmann-Boyce 2019). However, even with these treatments success rates are typically under $25 \%$, and half of those who try to quit do not use any support (Jackson 2019b). There remains an urgent need to identify new, effective and safer alternatives to cigarettes to reduce smoking prevalence.

\section{Description of the intervention}

Heated (or heat-not-burn) tobacco products (HTPs) are handheld devices that heat tobacco to a temperature that is high enough to produce a nicotine-infused aerosol, but too low to cause self-sustaining combustion. Many of the toxic and carcinogenic products of cigarette smoking are formed during combustion. HTPs are marketed as less harmful and as alternatives to conventional cigarettes because they attempt to avoid combustion (Mathers 2017). However, HTPs are relatively new to the market. The extent to which HTPs help people quit smoking is largely unknown, and their impact on youth uptake to smoking is contentious (Czoli 2020). It is therefore unclear what impact HTPs will have on smoking prevalence across the population.

The current HTP market is dominated by four products: 'IQOS' by Philip Morris International, 'glo' and 'iFuse' by British American Tobacco, and 'Ploom Tech' by Japan Tobacco International (WHO 2018). IQOS and glo produce aerosol by directly heating tobacco sticks, which resemble small cigarettes. Conversely, Ploom Tech and iFuse produce aerosol by heating a similar liquid to that found in e-cigarettes. This aerosol is then drawn through a bulb of tobacco to infuse it with flavour. Of these products, IQOS was the first to launch in 2014 in Japan and Italy, and it has since entered markets across Asia, Europe and America. Most recently, in 2019, the US Food and Drug Administration (FDA) permitted the sale of IQOS (FDA 2019) and in 2020 authorised their marketing as a modifiedexposure tobacco product (FDA 2020). At the time of writing, HTPs were most popular in Japan and the Republic of Korea; tobacco sticks for HTPs constituted $15.8 \%$ and $8.0 \%$ respectively of each country's tobacco market in 2018 (WHO 2018). Market research by Euromonitor estimates that HTPs had an increased share of the retail value of all nicotine or tobacco products between 2017 and 2018, which was similar to e-cigarettes globally (Euromonitor 2020)

\section{How the intervention might work}

Nicotine is the primary addictive compound in cigarettes, which causes people who quit smoking to experience withdrawal and cravings (West 2017). Like cigarettes, HTPs contain nicotine. They are expected to aid smoking cessation in a similar way to NRT and e-cigarettes: people can use them to relieve nicotine cravings without smoking cigarettes (Wadgave 2016). HTPs may also provide certain advantages over NRT. A limitation of NRT is that it poorly addresses the behavioural and sensory cues associated with cigarette smoking, such as repeated hand-to-mouth actions and the 'scratch' at the back of the throat when inhaling smoke. Evidence shows that denicotinised cigarettes reduce cravings and withdrawal symptoms among abstinent smokers, despite containing negligible levels of nicotine (Rose 2006). This suggests that these cues probably contribute to cigarette dependence. HTPs may more closely replicate these cues than NRT. Because HTP aerosol is delivered to the throat and lungs, nicotine absorption occurs more rapidly than from patches, gum or lozenges, which are absorbed through the skin or buccal mucosa (Simonavicius 2018). The speed with which nicotine is absorbed may be one of the key determinants of dependence (Benowitz 2009), so HTPs may provide a better replacement for cigarette smoking than NRT. Ecigarettes also deliver nicotine rapidly to the throat and possibly lungs (Wagener 2017; Hajek 2020) and, like HTPs, they mimic the hand-to-mouth actions of cigarette smoking. But only HTPs contain tobacco leaf, so their flavour may more closely resemble cigarette smoke (Poynton 2017), which may make them more attractive to smokers (Tompkins 2020). Moreover, in some countries, the sale of nicotine e-cigarettes is banned or heavily restricted (Dyer 2019). In such environments, HTPs may be the only consumer product available that delivers nicotine rapidly through a potentially less harmful medium than tobacco smoke.

A substantial proportion of people who use HTPs for smoking cessation may continue using these products for at least a year after they quit smoking, as is the case with e-cigarettes (Hajek 2019; Simonavicius 2020). We will refer to this as 'switching'. Encouraging people to switch from smoking cigarettes to using HTPs would only be beneficial if HTPs are less harmful to health or if HTPs eventually help people taper off nicotine entirely. The safety of HTPs to users depends on both the acute harm, measured by adverse and serious adverse events, and the long-term harm of repeated inhalation of damaging compounds in HTP aerosol. Biomarkers can be used to measure exposure to these harmful toxicants and carcinogens. Manufacturers of HTPs claim that the aerosol they produce contains significantly lower levels of toxicants than cigarette smoke and, as a result, that they have reduced risk potential or are less harmful (PMI 2018; BAT 2020). Two systematic reviews supported claims about lower toxicant levels, but found that most research into HTPs was funded through sources affiliated with the tobacco industry (Simonavicius 2018; Jankowski 2019). In addition, reduced exposure does not necessarily indicate reduced harm. The US FDA judged that there was sufficient evidence that iQOS reduced exposure to harmful chemicals (FDA 2020), but insufficient evidence on whether switching from smoking to HTPs reduces harm, such as pulmonary function or biomarkers linked to smoking-related harm (Glantz 2018; Moazed 2018). It is also the case that safety, especially of longer-term use, cannot be addressed with certainty until long-term cohort studies have collected sufficient data.

\section{Why it is important to do this review}

There is substantial variation between countries in their regulatory approaches to HTPs, and within countries across different nicotine products. In order for policymakers to regulate HTPs effectively and proportionately, there is a need for evidence to inform a judgement 
on their likely public health impact. The net impact of HTPs on public health will depend on a variety of factors. Three influential elements that could result in HTPs benefiting public health are if they increase smoking cessation, decrease smoking prevalence, and are less harmful than cigarette smoking. Conversely, HTPs could damage public health if they decrease smoking cessation, increase smoking prevalence, or are just as harmful as cigarette smoking.

The effect of HTP use on smoking prevalence will depend on whether they influence rates of attempted quitting among cigarette smokers, the proportion of these attempts that are successful, cigarette uptake among non-smokers, and relapse among people who had previously quit smoking. We are therefore not only interested in studies that report individual-level effects of HTPs on smoking cessation, but also those that estimate their populationlevel effects on smoking prevalence. This review will investigate upto-date evidence for both, using appropriate study types.

The growing popularity of HTPs means that people who smoke are likely to seek advice from practitioners who need to know whether HTPs are effective for smoking cessation and how their safety compares with cigarettes and other alternative nicotine products. If HTPs are found to be safe and effective for smoking cessation, they could add a novel treatment for cigarette addiction. Moreover, if there is evidence that an increase in HTP use prevalence is associated with a decrease in smoking prevalence, then this will help to guide the regulation of HTPs.

\section{O B JE C T IVES}

To evaluate the effectiveness and safety of HTPs for smoking cessation and the impact of HTPs on smoking prevalence.

\section{METHODS}

\section{Criteria for considering studies for this review}

\section{Types of studies}

We have divided the methods into the three subsections, representing the different objectives of the review:

\section{Effectiveness for smoking cessation}

We will include individual-level and cluster-RCTs to examine the effectiveness of HTPs for tobacco smoking cessation.

\section{Safety}

We will include individual-level, randomised cross-over and clusterRCTs to explore adverse and serious adverse events and biomarkers of toxicant and carcinogen exposure.

\section{Smoking prevalence}

We will include interrupted and multiple time-series studies to examine the population-level effect of HTPs on cigarettesmoking prevalence. Smoking cessation interventions may not be representative of the way most people use HTPs, without support from a researcher or trained specialist. Moreover, even if HTPs encourage smoking cessation among those trying to quit, their impact on smoking prevalence depends on how they affect smoking initiation and the number of people who make a quit attempt. We will use time-series studies to assess how changes in HTP prevalence are associated with changes in smoking prevalence, with the limitation that associations might not reflect causal effects.

We will include studies regardless of language or status of publication.

\section{Types of participants}

\section{Effectiveness and safety}

We will include adults, defined as current cigarette smokers by the study, at the time of enrolment.

\section{Smoking prevalence}

We will not restrict by participant characteristics, as we are interested in population-level data. We will focus on any individuals who report on their smoking status or consumption and HTP use or consumption, measured by survey or by record of sales.

\section{Types of interventions}

HTPs, defined as hand-held devices that heat tobacco to a temperature that is high enough to produce a nicotine-infused aerosol but too low to cause self-sustaining combustion. HTPS differ from e-cigarettes in that they produce aerosol by heating compressed tobacco leaf rather than a liquid that is infused with nicotine concentrate.

\section{Effectiveness and safety}

We are interested in studies that compare HTPs, or the addition of HTPs, to no treatment (i.e. continued tobacco smoking), placebo or any other smoking cessation treatment, including nicotine replacement therapy (NRT), electronic cigarettes, varenicline, bupropion, and behavioural support. HTPs can be provided in addition to any other smoking cessation treatment, providing there is equivalent provision for the control group. We will only include studies where participants were instructed to stop smoking combustible cigarettes.

\section{Smoking prevalence}

We are interested in studies that estimate the extent to which changes in the prevalence of HTP use are associated with changes in the prevalence of cigarette smoking, after adjusting for other influences that could affect changes in the prevalence of smoking at the population level.

\section{Types of outcome measures}

\section{Primary outcomes}

\section{Effectiveness}

- Tobacco smoking cessation at the longest follow-up point available, using intention-to-treat and biochemically-verified abstinence where possible. While HTPs contain tobacco, they are designed to heat the tobacco and attempt to avoid or minimise combustion and smoke. HTP use will therefore not be classified as tobacco smoking. We will restrict the review to studies which report abstinence at four-week follow-up or longer. We will use the strictest definition of abstinence recorded, i.e. prolonged or continuous abstinence over point prevalence, and biochemically-verified over selfreported abstinence.Typically, Cochrane Tobacco Addiction Group reviews only include data on smoking cessation at six months or longer. We will include short-term outcomes in this 
review because we anticipate a paucity of longer-term data. In future updates, as and when more data become available, we may change the inclusion criteria accordingly.

\section{Safety}

- Number of people reporting adverse events (AEs) and serious adverse events (SAEs).

\section{Smoking prevalence}

- Change in the prevalence of cigarette smoking, measured as the proportion of people in a given locality that regularly smoke cigarettes or other combustible tobacco products, over a defined time period. We will include cigarette sales as a proxy for prevalence and measured as the number of cigarettes sold in a given locality over a given time period.

\section{Secondary outcomes}

All secondary outcomes are measures of safety. We will only include studies which report safety outcomes at one-week followup or longer.

- Biomarkers of toxicant and carcinogen exposure. We will include measures of exposure to tobacco-specific N-nitrosamines, polycyclic aromatic hydrocarbons, volatile organic compounds, and carbon monoxide (see Appendix 1 for details).

- Biomarkers of harm. We will include measures of lung function (FEV1, FVC and FEV1/FVC), blood pressure, heart rate, heart rate variability, and blood oxygen saturation.

\section{Search methods for identification of studies}

\section{Electronic searches}

We will search the following databases:

- Cochrane Central Register of Controlled Trials;

- Cochrane Tobacco Addiction Group's Specialised Register (for details of how this register is populated see the Cochrane Tobacco Addiction Group's website);

- MEDLINE (OVID SP);

- Embase (OVID SP);

- PsycINFO (OVID SP);

- Business Source Complete;

- Factiva;

- Clinicaltrials.gov;

- The WHO International Clinical Trials Registry Platform (ICTRP) (apps.who.int/trialsearch/).

We will restrict the search to studies published since 2008, three years before the first internet searches for HTPs began (Google Trends 2020).

The search terms will be:

heated tobacco OR carbon-heated tobacco OR heat-not-burn OR heat not burn OR tobacco heating system\$ OR tobacco heating device OR tobacco heating product\$ OR tobacco vapor product $\$$ OR tobacco vapour product\$. We will also search for the term smoking AND (iqos OR glo OR ploom OR ifuse OR fuse OR pulse OR teeps OR pax OR mok OR lil OR iuoc OR htp OR thp OR ths OR chtp).
As we are only interested in studies that use human subjects, we will exclude those with the terms animal\$ OR mice OR rat\$ OR in vitro OR in silico OR in vivo in their title.

\section{Searching other resources}

We will search the reference lists of eligible studies found in the literature search.

In order to identify government reports and in-press or unpublished studies, we will contact tobacco control charities and authors of published research or trial protocols. We will use the searches of Clinicaltrials.gov and the ICTRP detailed above to identify trial registry records.

\section{Data collection and analysis}

\section{Selection of studies}

Two review authors will independently pre-screen titles and abstracts of articles identified in the search, using a screening checklist. We will resolve disagreements through discussion or referral to a third review author. We will conduct screening using Covidence software (Covidence).

Two review authors will independently screen the full text of articles that pass pre-screening, with a third review author to resolve any disagreements that are not resolved through discussion.

\section{Data extraction and management}

We will produce two custom data extraction forms: one for effectiveness and safety, and the other for smoking prevalence. Both will include:

- Author;

- Study design;

- Study dates;

- Date of publication;

- Inclusion and exclusion criteria;

- Setting;

- Summary of study population characteristics;

- Time points at which outcomes were assessed;

- Source of study funding;

- Author's declarations of interest;

- Additional information.

Effectiveness and safety forms will also include:

- Summary of intervention and control conditions, including HTP product and intensity of behavioural support available, where relevant;

- Smoking cessation definition used;

- Smoking cessation outcomes;

- Form of biochemical validation used, where relevant;

- AEs and SAEs;

- Biomarkers of polycyclic aromatic hydrocarbon exposure (1hydroxypyrene) at baseline and longest follow-up available;

- Biomarkers of carbon monoxide exposure (exhaled carbon monoxide or carboxyhaemoglobin) at baseline and longest follow-up point available; 
- Biomarkers of exposure to the volatile organic compounds acrolein, lead, cadmium, and butadiene (3-HPMA, lead, cadmium, and MHBMA3 respectively) at baseline and longest follow-up point available;

- Biomarkers of tobacco-specific N-nitrosamine (TSNA) exposure (NNAL) at baseline and longest follow-up point available;

- Lung function (measured using FEV1, FVC and FEV1/FVC);

- Blood pressure;

- Heart rate;

- Blood oxygen saturation.

Smoking prevalence forms will also include:

- Coefficient and standard error for change in the trend following intervention in prevalence or sales;

- Coefficient and standard error for step-level change in prevalence or sales;

- Coefficient and standard error for changes between cigarette prevalence or sales and heated tobacco product prevalence or sales;

- Statistical method used;

- Covariates included in model;

- Temporal granularity (e.g. weekly, monthly, annual);

- Time when heated tobacco products entered the market;

- Total time points at which outcomes were assessed;

- Pre-intervention time points;

- Post-intervention time points;

- Stationarity;

- Seasonality;

- Autocorrelation;

- Lags;

- Model fit.

Two review authors will independently extract data from included studies. When discrepancies cannot be resolved through discussion, we will refer to a third review author. We will contact authors of included studies if additional information is needed.

\section{Assessment of risk of bias in included studies}

\section{Effectiveness and safety}

Two review authors will independently assess risks of bias for all included RCTs using the Cochrane 'Risk of bias' tool (ROB). The version we use ( 1 or 2 ) will depend on software availability at the time of data extraction. Regardless of which tool we use, we will follow the guidance as set out in the Cochrane Handbook for Systematic Reviews of Iterventions (Handbook) to evaluate the appropriate domains. For ROB 1 these are sequence generation, allocation concealment, blinding of participants, personnel and outcome assessment, incomplete outcome data, and selective reporting, as well as other sources of bias (Higgins 2011). However, if version 2 is deemed feasible, we will evaluate studies within the following domains: bias arising from the randomisation process; bias due to deviations from intended interventions; bias due to missing outcome data; bias in measurement of the outcome; bias in selection of the reported result (Higgins 2020).

\section{Smoking prevalence}

Two review authors will independently assess risks of bias for included time-series studies using the ROBINS-I tool (Sterne 2016).

\section{Measures of treatment effect}

\section{Effectiveness and safety}

We will calculate risk ratios (RRs) and $95 \%$ confidence intervals (Cls) for binary outcomes, such as smoking cessation.

We will calculate mean differences (MDs) and the corresponding $95 \% \mathrm{Cls}$, between the intervention and control groups in the mean change from baseline to follow-up for continuous safety data.

For smoking cessation, we will use the longest follow-up data reported, and for safety outcomes we will assess data at all time points. In both cases we will calculate treatment effects on an intention-to-treat basis.

\section{Smoking prevalence}

For interrupted time-series studies, the treatment effect will be reflected by the step change and the change in trends in smoking prevalence or cigarette sales following the introduction of HTPs to the market, after controlling for confounding variables.

For multiple time-series studies, the treatment effect will be the association between HTP prevalence and smoking prevalence or cigarette sales, after controlling for confounding variables. It is likely that the variables will have been log-transformed to address non-stationarity and for easier interpretation. The resulting coefficient would describe the percentage change in cigarette smoking prevalence associated with a $1 \%$ change in HTP prevalence.

\section{Unit of analysis issues}

\section{Effectiveness and safety}

For RCTs with more than two intervention arms, we will combine data from all relevant intervention conditions where HTPs were offered, where appropriate. If it is not appropriate to pool the intervention arms then we will split the control arm to act as a comparator to each separate intervention arm. For RCTs with more than two control arms, we will not combine data from each of these arms, and we will choose the most appropriate comparator. For cluster-RCTs, we will attempt to extract an estimate of the effect that accounts for the cluster design of the study. Where this is not reported, we will attempt to perform the correct analysis if required data are available.

\section{Dealing with missing data}

\section{Effectiveness}

When assessing smoking cessation, we will assume that people with missing data at follow-up have not stopped smoking, as is common in the field.

\section{Safety}

When assessing AEs and SAEs, we will calculate the proportion of those available at follow-up who experienced an event, rather than the proportion of people who were randomised. When assessing biomarkers, we will remove participants with missing follow-up data from the analysis. 


\section{Smoking prevalence}

We do not anticipate problems with missing data in reported timeseries studies.

\section{Assessment of heterogeneity}

To assess whether to conduct meta-analyses, we will consider the characteristics of included studies to identify substantial clinical or methodological heterogeneity. If we deem the data to be sufficiently homogeneous to combined them meaningfully, we will assess statistical heterogeneity using the $\mathrm{I}^{2}$ statistic. If $\mathrm{I} 2$ is greater than $50 \%$, we will report substantial heterogeneity.

\section{Assessment of reporting biases}

If we deem it appropriate to pool 10 or more studies in any analysis we will assess reporting bias using funnel plots. The greater the asymmetry in the plots, the higher the risk of reporting bias.

\section{Data synthesis}

\section{Effectiveness}

The primary outcome of smoking cessation provides dichotomous data. Following the standard methods of the Cochrane Tobacco Addiction Group, we will combine RRs and $95 \% \mathrm{Cls}$ from individual studies using a Mantel-Haenszel random-effects model, to calculate pooled overall RRs with $95 \% \mathrm{Cls}$.

\section{Safety}

For dichotomous safety outcomes (i.e. AEs and SAEs) we will combine RRs and $95 \%$ Cls from individual studies using a MantelHaenszel random-effects model, to calculate pooled overall RRs with $95 \%$ Cls.

For continuous safety outcomes measuring biomarkers, we will pool the MDs or standardised mean differences (SMDs) and measures of variance of individual studies using a generic inverse variance random-effects model.

\section{Smoking prevalence}

We will calculate pooled estimates and their standard errors using a random-effects model for each of three coefficients: step change in smoking prevalence or cigarette sales following the introduction of HTPs; change in these trends after the introduction; and changes associated with changes in prevalence or sale of HTPs. For timeseries studies with notably different time periods (for example, weekly versus annual), we will be unable to pool between studies.

\section{Subgroup analysis and investigation of heterogeneity}

We plan to undertake subgroup analyses to investigate differences by:

- Intensity of behavioural support provided;

- characteristics of HTP device (e.g. model used).

\section{Sensitivity analysis}

We will carry out sensitivity analyses where we remove studies:

- judged to be at high risk of bias for at least one domain;

- where the study is funded by (or authors have received funding from) the tobacco industry;

- with a minimum length of follow-up less than four weeks (safety outcomes only).

We will also carry out sensitivity analyses where we:

- only include participants who exclusively used the product they were assigned to (safety outcomes only);

- only classify participants as HTP users if they use their product daily (smoking prevalence only).

\section{'Summary of findings' tables and GRADE}

We will create a 'Summary of findings' table using GradePro for all primary outcomes (GRADEpro GDT), following the guidelines in Cochrane Handbook of Systematic Reviews of Interventions 6.1 (Schünemann 2020). We will use the five GRADE considerations (risk of bias, inconsistency, imprecision, indirectness and publication bias) to assess the certainty of the body of evidence for each of these outcomes.

\section{ACK N OWLEDGEMENTS}

We gratefully acknowledge peer review by K. Michael Cummings, $\mathrm{PhD}, \mathrm{MPH}$, Medical University of South Carolina, USA and Sophie Braznell, Tobacco Control Research Group, Department for Health, University of Bath, Bath, UK, and consumer review by Sandra Wilcox. We would like to thank the following organisations who provided salary or studentship support to the authors of this review protocol: Public Health England, NIHR Maudsley Biomedical Research Centre, UK Prevention Research Partnership, and Cancer Research UK. The views and opinions expressed therein are those of the authors and do not necessarily reflect those of these organisations. 


\section{REFERE N C E S}

\section{Additional references}

\section{BAT 2020}

British American Tobacco. The science behind glo. www.bat-science.com/groupms/sites/BAT_B9JBW3.nsf/ vwPagesWebLive/DOBA2J7K 2020 (accessed 15 November 2020).

\section{Benowitz 2009}

Benowitz NL. Pharmacology of nicotine: addiction, smokinginduced disease, and therapeutics. Annual Review of Pharmacology and Toxicology 2009;49(1):57-71. [DOI: 10.1146/ annurev.pharmtox.48.113006.094742]

\section{Cahill 2016}

Cahill K, Lindson-Hawley N, Thomas KH, Fanshawe TR, Lancaster T. Nicotine receptor partial agonists for smoking cessation. Cochrane Database of Systematic Reviews 2016, Issue 5. Art. No: CD006103. [DOI: 10.1002/14651858.CD006103.pub7]

\section{Chang 2017}

Chang CM, Edwards SH, Arab A, Del Valle-Pinero AY, Yang L, Hatsukami DK. Biomarkers of tobacco exposure: summary of an FDA-sponsored public workshop. Cancer Epidemiology, Biomarkers \& Prevention 2017;26(3):291-302. [DOI: 10.1158/1055-9965.EPI-16-0675]

\section{Covidence [Computer program]}

Veritas Health Innovation Covidence. Version accessed 10 May 2020. Melbourne, Australia: Veritas Health Innovation. Available at covidence.org.

\section{Czoli 2020}

Czoli CD, White CM, Reid JL, Oconnor RJ, Hammond D. Awareness and interest in IQOS heated tobacco products among youth in Canada, England and the USA. Tobacco Control 2019;29(1):89-95. [DOI: 10.1136/tobaccocontrol-2018-054654]

\section{Drope 2018}

Drope J, Schluger N, Cahn Z, Drope J, Hamill S, Islami F, et al. The Tobacco Atlas. 6 edition. Atlanta: American Cancer Society and Vital Strategies, 2018.

\section{Dyer 2019}

Dyer 0 . India bans e-cigarettes by executive order. BMJ 2019;366:i5649. [DOI: doi:10.1136/bmj.l5649]

\section{Euromonitor 2020}

Euromonitor International. Smokeless tobacco, e-vapour products and heated tobacco in world. Euromonitor Passport 2020.

\section{FDA 2019}

US Food and Drug Administration. FDA permits sale of IQOS Tobacco Heating System through premarket tobacco product application pathway. www.fda.gov/news-events/pressannouncements/fda-permits-sale-iqos-tobacco-heatingsystem-through-premarket-tobacco-product-applicationpathway 2019.

\section{FDA 2020}

US Food and Drug Administration. FDA Authorizes Marketing of IQOS Tobacco Heating System with 'Reduced Exposure' Information. www.fda.gov/news-events/press-announcements/ fda-authorizes-marketing-iqos-tobacco-heating-systemreduced-exposure-information 2020.

\section{Glantz 2018}

Glantz SA. PMI's own in vivo clinical data on biomarkers of potential harm in Americans show that IQOS is not detectably different from conventional cigarettes. Tobacco Control 2018;27:9-12. [DOI: 10.1136/tobaccocontrol-2018-054413]

\section{Google Trends 2020}

Google Trends. Worldwide internet searches for "heat-notburn" from 2004-2020. trends.google.com/trends/explore? date=all\&q=heat-not-burn 2020 .

\section{GRADEpro GDT [Computer program]}

McMaster University (developed by Evidence Prime) GRADEpro GDT. Version accessed 10 May 2020. Hamilton (ON): McMaster University (developed by Evidence Prime). Available at gradepro.org.

\section{Hajek 2019}

Hajek P, Phillips-Waller A, Przulj D, Pesola F, Myers Smith K, Bisal $N$, et al. A randomized trial of e-cigarettes versus nicotinereplacement therapy. New England Journal of Medicine 2019;380(7):629-37. [DOI: 10.1056/NEJMoa1808779]

\section{Hajek 2020}

Hajek P, Pittaccio K, Pesola F, Myers Smith K, Phillips-Waller A, Przulj D. Nicotine delivery and users' reactions to Juul compared with cigarettes and other e-cigarette products. Addiction 2020;115(6):1141-8. [DOI: 10.1111/add.14936]

\section{Hartmann-Boyce 2018}

Hartmann-Boyce J, Chepkin SC, Ye W, Bullen C, Lancaster T. Nicotine replacement therapy versus control for smoking cessation. Cochrane Database of Systematic Reviews 2018, Issue 5. Art. No: CD000146. [DOI: 10.1002/14651858.CD000146.pub5]

\section{Hartmann-Boyce 2019}

Hartmann-Boyce J, Hong B, Livingstone-Banks J, Wheat H, Fanshawe TR. Additional behavioural support as an adjunct to pharmacotherapy for smoking cessation. Cochrane Database of Systematic Reviews 2019, Issue 6. Art. No: CD009670. [DOI: 10.1002/14651858.CD009670.pub4]

\section{Hedblad 2005}

Hedblad B, Ögren M, Engström G, Wollmer P, Janzon L. Heterogeneity of cardiovascular risk among smokers is related to degree of carbon monoxide exposure. Atherosclerosis 2005;179(1):347-64. [DOI: 10.1016/ j.atherosclerosis.2004.10.005]

\section{Higgins 2011}

Higgins JP, Altman DG, Gøtzsche PC, Jüni P, Moher D, Oxman AD, et al. The Cochrane Collaboration's tool for 
assessing risk of bias in randomised trials. BMJ 2011;343:d5928. [DOI: 10.1136/bmj.d5928]

\section{Higgins 2020}

Higgins JP, Savović J, Page MJ, Elbers RG, Sterne JA. Chapter 8: Assessing risk of bias in a randomized trial. In: Higgins JPT, Thomas J, Chandler J, Cumpston M, Li T, Page MJ, et al (editors). Cochrane Handbook for Systematic Reviews of Interventions version 6.1 (updated September 2020). The Cochrane Collaboration, 2020. Available from www.training.cochrane.org/ handbook.

\section{Holford 2014}

Holford T, Meza R, Warner K, Meernik CE, Jeon JM, Suresh H, et al. Tobacco control and the reduction in smoking-related premature deaths in the United States, 1964-2012. JAMA 2014;311(2):164-71.

\section{IARC 2012}

IARC Working Group on the Evaluation of Carcinogenic Risks to Humans. Personal habits and indoor combustions: a review of human carcinogens.. IARC Monographs on the Evaluation of Carcinogenic Risks to Humans 2012. [PMID: 23193840]

\section{Jackson 2019a}

Jackson SE, McGowan JA, Ubhi HK, Proudfoot H, Shahab L, Brown J, et al. Modelling continuous abstinence rates over time from clinical trials of pharmacological interventions for smoking cessation. Addiction 2019;114(5):787-97. [DOI: 10.1111/ add.14549]

\section{Jackson 2019b}

Jackson SE, Kotz D, West R, Brown J. Moderators of real-world effectiveness of smoking cessation aids: a population study. Addiction 2019;114(9):1627-38. [DOI: 10.1111/add.14656]

\section{Jankowski 2019}

Jankowski M, Brożek GM, Lawson J, Skoczyński S, Majek P, Zejda JE. New ideas, old problems? Heated tobacco products - a systematic review. International Journal of Occupational Medicine and Environmental Health 2019;32(5):595-634. [DOI: 10.13075/ijomeh.1896.01433]

\section{Kim 2013}

Kim KH, Jahan SA, Kabir E, Brown RJ. A review of airborne polycyclic aromatic hydrocarbons (PAHs) and their human health effects. Environment International 2013;60:71-80. [DOI: 10.1016/j.envint.2013.07.019]

\section{Mathers 2017}

Mathers A, Schwartz R, O'Connor S, Fung M, Diemert L. Marketing IQOS in a dark market. Tobacco Control 2017;28:237-8. [DOI: 10.1136/tobaccocontrol-2017-054216]

\section{Moazed 2018}

Moazed F, Chun L, Matthay MA, Calfee CS, Gotts J. Assessment of industry data on pulmonary and immunosuppressive effects of IQOS. Tobacco Control 2018;27:20-25. [DOI: 10.1136/ tobaccocontrol-2018-054296]

\section{PMI 2018}

Phillip Morris International. The Difference between Switching to IQOS and Continuing to Smoke Cigarettes. www.pmiscience.com/discover/news/the-difference-betweenswitching-to-iqos-and-continuing-to-smoke-cigarettes 2018.

\section{Poynton 2017}

Poynton S, Sutton J, Goodall S, Margham J, Forster M, Scott K, et al. A novel hybrid tobacco product that delivers a tobacco flavour note with vapour aerosol (Part 1): Product operation and preliminary aerosol chemistry assessment. Food and Chemical Toxicology 2017;106:522-32. [DOI: 10.1016/j.fct.2017.05.022]

\section{Rose 2006}

Rose J. Nicotine and nonnicotine factors in cigarette addiction. Psychopharmacology 2006;184:274-285. [DOI: 10.1007/ s00213-005-0250-x]

\section{Schettgen 2008}

Schettgen T, Musiol A, Kraus T. Simultaneous determination of mercapturic acids derived from ethylene oxide (HEMA), propylene oxide (2-HPMA), acrolein (3-HPMA), acrylamide (AAMA) and $\mathrm{N}, \mathrm{N}$-dimethylformamide (AMCC) in human urine using liquid chromatography/tandem mass spectrometry. Rapid Communications in Mass Spectrometry 2008;22(17):2629-38. [DOI: 10.1002/rcm.3659]

\section{Schünemann 2020}

Schünemann HJ, Higgins JP, Vist GE, Glasziou P, Akl EA, Skoetz N, et al. Chapter 14: Completing 'Summary of findings' tables and grading the certainty of the evidence. In: Higgins JP, Thomas J, Chandler J, Cumpston M, Li T, Page MJ, et al (editors). Cochrane Handbook for Systematic Reviews of Interventions version 6.1 (updated September 2020). The Cochrane Collaboration, 2020. Available from www.training.cochrane.org/ handbook.

\section{Shahab 2017}

Shahab L, Goniewicz M, Blount B, Brown J, McNeill A, Alwis K, et al. Nicotine, carcinogen and toxicant exposure in long-term e-cigarette and nicotine replacement therapy users: a crosssectional study. Annals of Internal Medicine 2017;166(6):390-400. [DOI: 10.7326/M16-1107]

\section{Simonavicius 2018}

Simonavicius E, McNeill A, Shahab L, Brose LS. Heatnot-burn tobacco products: a systematic literature review. Tobacco Control 2018;28(5):582-94. [DOI: 10.1136/ tobaccocontrol-2018-054419]

\section{Simonavicius 2020}

Simonavicius E, McNeill A, Brose LS. Transitions in smoking and nicotine use from 2016 to 2017 among a UK cohort of adult smokers and ex-smokers. Drug and Alcohol Review 2020 [epub ahead of print]. [DOI: 10.1111/dar.13063]

\section{Sterne 2016}

Sterne J, Hernán M, Reeves B, Savović J, Berkman N, Viswanathan M, et al. ROBINS-I: A tool for assessing risk of bias in non-randomised studies of interventions. $B M J$ 2016;355:4919. [DOI: 10.1136/bmj.i4919] 


\section{Tompkins 2020}

Tompkins CN, Burnley A, McNeill A, Hitchman SC. Factors that influence smokers' and ex-smokers' use of IQOS: a qualitative study of IQOS users and ex-users in the UK. Tobacco Control 2020:tobaccocontrol-2019-055306. [DOI: 10.1136/ tobaccocontrol-2019-055306]

\section{Wadgave 2016}

Wadgave U, Nagesh L. Nicotine replacement therapy: an overview. International Journal of Health Science 2016;32(8):425-35. [DOI: 10.12816/0048737]

\section{Wagener 2017}

Wagener TL, Floyd EL, Stepanov I, Driskill LM, Frank SG, Meier E, et al. Have combustible cigarettes met their match? The nicotine delivery profiles and harmful constituent exposures of second-generation and third-generation electronic cigarette users. Tobacco Control 2017;26(e1):23-8. [DOI: 10.1136/

tobaccocontrol-2016-053041]

\section{West 2017}

West R. Tobacco smoking: health impact, prevalence, correlates and interventions. Psychology \& Health 2017;32(8):1018-36. [DOI: 10.1080/08870446.2017.1325890]

\section{WHO 2018}

World Health Organisation. Heated Tobacco Products (HTPs) Market Monitoring Information. www.who.int/tobacco/ publications/prod_regulation/htps-marketing-monitoring/en/ 2018.

\section{Yeager 2016}

Yeager P, Kushman M, Chemerynski S, Weil R, Fu X, White M, et al. Proposed mode of action for acrolein respiratory toxicity associated with inhaled tobacco smoke. Toxicological Sciences 2016;151(2):347-64. [DOI: 10.1093/toxsci/kfw051]

\section{APPENDICES}

\section{Appendix 1. Biomarkers of toxicant and carcinogen exposure}

Where available, we will report on exposure to the following toxicants and carcinogens, using the biomarkers listed below:

- Tobacco-specific N-nitrosamine (TSNA) exposure (measured using the biomarker urinary NNAL). Several TSNAs are group 1 or $2 \mathrm{~A}$ carcinogens, implicated in the increased incidence of cancer among smokers ( IARC 2012 ); 4-(methylnitrosamino)-1-(3-pyridyl)-1butanol (NNAL) is the most widely investigated biomarker of TSNA exposure (Chang 2017); and NNAL is found in high quantities among cigarette smokers, but very low quantities among NRT and e-cigarette users (Shahab 2017). It therefore also gives an indication of the safety profile of HTPs when compared with other smoking cessation aids.

- Polycyclic aromatic hydrocarbon exposure (measured using the urinary biomarkers 1-hydroxypyrene and 1- and 2hydroxynaphthalene). Polycyclic aromatic hydrocarbons are produced though incomplete combustion of organic compounds, as occurs through cigarette smoking. Exposure to these compounds is linked to cancers along with DNA, kidney, and liver damage (Kim 2013).

- Exposure to the volatile organic compounds acrolein, heavy metals, and butadiene (measured using the biomarkers 3-HPMA, heavy metals, and MHBMA3 respectively). Acrolein is implicated as the key compound associated with smoking-induced respiratory disease (Yeager 2016). 3-HPMA is a widely used urinary biomarker of acrolein exposure (Schettgen 2008). Carcinogenic heavy metals, like lead and cadmium, are present in cigarette smoke (IARC 2012). Butadiene is a group 1 carcinogen.

- Carbon monoxide exposure (measured using exhaled carbon monoxide or carboxyhaemoglobin in blood). High exposure to carbon monoxide among sole HTP users would indicate that the tobacco in HTPs has undergone pyrolysis or combustion. Carbon monoxide exposure is linked to the increased risk of cardiovascular disease among smokers (Hedblad 2005).

\section{HISTORY}

Protocol first published: Issue 11, 2020

\section{CONTRIBUTIONS OFAUTHORS}

All authors contributed to the writing and editing of this protocol.

\section{DECLARATIONS OF INTEREST}

ES has a PhD studentship funded by the NIHR Maudsley Biomedical Research Centre. This is not deemed to be a conflict of interest. HTB holds a studentship funded by Public Health England. This is not deemed to be a conflict of interest.

JB has undertaken research and consultancy for manufacturers of smoking cessation medications (Pfizer and Johnson \& Johnson). JHB has no conflicts of interest to declare. 
LK salary is funded by the UK Prevention Research Partnership, an initiative funded by UK Research and Innovation Councils, the Department of Health and Social Care (England), and the UK devolved administrations and leading health research charities. This is not deemed to be a conflict of interest.

LS has received unrestricted funding from Pfizer to study smoking cessation treatments and has consulted for Alantis Healthcare - writing a framework for medication to treat smoking-related disease.

LB has received funding from Public Health England, Cancer Research UK and Heart Research UK. These are not deemed to be conflicts of interests.

SJ has received funding from Cancer Research UK and the Economic and Social Research Council. These are not deemed to be conflicts of interests.

None of the authors have received cash or kind, hospitality, or any subsidy from manufactures of tobacco products or electronic cigarettes.

\section{SOURCES OFSUPPORT}

\section{Internal sources}

- University College London, UK

Provides salary, office space, and/or library resources for HTB, SJ, LS, LK, and JB.

- Kings College London, UK

Provides salary, office space, and/or library resources for LB and ES.

- University of Oxford, UK

Provides salary, office space, and library resources for JHB.

\section{External sources}

- Public Health England, UK

Provides PhD studentship funding for HTB.

- Cancer Research UK, UK

Provides salary support for SJ.

- NIHR Maudsley Biomedical Research Centre, UK

Provides PhD studentship funding for ES.

- UK Prevention Research Partnership, UK

Provides salary support for LK. 